\title{
A Case of Nursing Experience of Taking Care of Creutzfeldt-Jakob Disease Patient in China
}

Wang Feifei*

Faculty of Public Health, University of Debrecen, Hungary

*Corresponding author: Wang Feifei, Faculty of Public Health, University of Debrecen, Hungary, Tel: 06702833412; E-mail: wffnurse@163.com

Received date: Feb 17, 2016, Accepted date: Mar 29, 2016, Published date: Apr 06, 2016

Copyright: (c) 2016 Feifei W. This is an open-access article distributed under the terms of the Creative Commons Attribution License, which permits unrestricted use, distribution, and reproduction in any medium, provided the original author and source are credited.

\begin{abstract}
Human prion disease is known as new variant Creutzfeldt-Jakob disease (CJD), prion protein is not virus, but a special protein which is infectious (protinactious infectious particles, PRION). Creutzfeldt-Jakob disease is a group of infectious prion protein caused by sporadic, dominantly inherited, transmissible neurodegenerative disease, which is characterized by pathologic spongy degeneration of the brain. Previously authors have published their idea in detail to prion protein disease. Detailed criteria of protecting medical staff and patients for this kind of disease was illustrated. In China, many hospitals have limited equipment and lacking knowledge of rare disease.

We treated a suspicious CJD patient and diagnosed with CJD after professional test. We took many measures to protect people who have direct or indirect contact with the patient and to prevent the transmission of virus. Firstly, we provide protection knowledge for health care workers and patient's families. Secondly, we made strict infection and isolation for environment and instruments. Thirdly, cut off the transmission and other factors to prevent the spread of disease. We are successfully got our goal that nobody was infected during the hospitalization. Creutzfeldt-Jakob disease has a high infection possibility, it is apparent that positive and effective protection management is needed. Health education has an important significance for preventing Creutzfeldt-Jakob disease infection. Here is report presenting how we took care of the CJD patient.
\end{abstract}

Keywords: Creutzfeldt-Jakob disease; Nursing measures; Protection methods; Transmission; Nursing manage

\section{Introduction}

Human prion disease is known as new variant Creutzfeldt-Jakob disease (CJD), prion protein is not virus, but a special protein which is infectious (protinactious infectious particles, PRION) [1-4]. Creutzfeldt-Jakob disease is a group of infectious prion protein caused sporadic, dominantly inherited, transmissible neurodegenerative disease, which is characterized by pathologic spongy degeneration of the brain [5]. CJD is more likely to happen to women who are 40-80 years old, and it has a long incubation period, short course, patients usually died within 1 year after the onset of symptoms [6]. CJD is a transmissible neurodegenerative disease, early diagnosis is difficult, it is likely to spread, and there is no effective treatment. Sperling [7] defined this disease as a rare, sporadic disease with no recognizable pattern of transmission or as a familial disease associated with prion protein gene mutations, therefore, high degree of emphasis on Creutzfeldt-Jakob disease is especially important. My department treated one case of CJD patients in 2015. Nursing procedures are showed as follows:

\section{Background Information}

Patient, female, 55 years old, Han nationality, from Shaanxi province. Patient had headache without obvious incentive on 5th November 2014, and followed with persistent pain at top of the head, blurred vision, staggering, hearing loss, and head MRI shows: lacunar infarction. She did not feel better at local hospital for one week. She came to our hospital and took treatment in outpatient for a week from 2nd December. But the symptoms become progressively worse, limbs stiff, unable to walk under the arm. The outpatient office delivered the patient to my department with vertigo syndrome, cerebral diagnosis on 11th December. Physical examination: clear consciousness, slurred speech, abilities in thinking, comprehension, orientation, memory and computing power decreased; bilateral pupillary are same and normal, light reaction was sensitive, but binocular vision decreased; limb muscle strength 5 , muscle tension increased, pointing trials, rotation trials and knee trials were in coordination precisely. After consenting with patient and her families, they agreed to take brain tissue for the aim of laboratory examination, diagnosed as: Creutzfeldt-Jakob disease.

\section{Creutzfeldt-Jakob Disease Patient Protection Measures}

\section{Self-protection of medical staff}

CJD is a fatal infectious disease. MEDICAL Staff should pay attention to physical breakage, avoid direct contact of the patient's blood and body fluids come from skin, conjunctiva, the mucous membrane, cerebrospinal fluid and other. Nurses' work is to do various operations such as injection, cannula, oral care, taking specimens, etc. They are prone to be infected. Take an example, when patient is twitching, nurse must stop dialing needle, in consideration of avoiding needle-stick to the skin. From the perspective of prevention, medical staff and laboratory workers should wash their hands after contact with patients, disinfect if necessary. 
Page 2 of 3

\section{Prevention and care for paroxysmal myoclonic}

Create a good environment for the ward: Create a good environment to keep the ward quiet, soft light, and do not use direct light to shine the patient's head. Avoid any sound and lights that can cause myoclonus.

Precautions of Care operations: In order to avoid myoclonus caused by stimulation treatment operation, nurses need to explain to patient before the operation. All operations should better be finished over a short period of time as much as possible to shorten the time in order guarantee not to be stimulated the patient. Care givers should follow the "4 lightly" (talking lightly, walking lightly, closing door lightly, operating lightly), thereby reducing myoclonic seizures.

Security for patients: Good bed protection measures and other security measures to prevent the occurrence of falls, accidents such as falling out of bed are essentially do good to patient. Bedside protection is necessary when Myoclonus, muscle tonic is attacking. Restraint belt is forbidden in case of fracture, traction injury.

\section{Infection prevention and care}

Prevention of lung infection: First, keep efficient air circulation in the ward, ventilation regularly. Attention should be paid that keeping the patient warm and preventing colds is a basic job. Try to reduce the number of visits, and air sterilization 2 times/day. Good oral care is needed, at the same time, to observe oral mucosa conditions. Back massage for patient every 2-3 hours, and stimulate patient to have effective cough and give expectoration, and then give inhalation. After those effective measures, the patient was without lung infection during hospitalization. This disease and other chronic disease like CVD require that there be a working patient-provider "partnership" that involves effective treatment within an integrated system of collaborative care which includes self-management education and follow up [8]. Leonardo lead a project showed that care manager who connect the role of "partnership" between patient and medical professionals have a positive impact and effective promotion [9]. In China, nurses usually take the role of care manager to reach the goal of Qualitative Nursing Service [10].

Prevent urinary tract infections: Give catheterization, bladder irrigation to the patient; the flushing fluid is $0.02 \%$ Nitrofurazone. Keeping the perineum clean and dry makes it much easier to reach the goal of preventing urinary tract infections. After those effective measures, the patient did not get Urinary tract infections during hospitalization.

\section{Prevent skin ulcer}

If a patient stayed in bed for a long time and has little movement, she is prone to get sores, sore is easily caused by pressure. Change clothes for patient every day, and turn over body for patient, adding bony protrusion massage 2 hours each time, 3 times a day. Give liquid nutritious food such as milk to the patient through her nose, and other liquid nutritious we use is $25 \%$ of medium/long-chain fat emulsion injection (C8-24), and Compound Amino Acid Injection (18AA-VII) by intravenous infusion to ensure sufficient daily energy. After those effective care measures, the patient without pressure sores during the hospitalization.

\section{Intelligent and functional decline of the patient}

Talk with patient initiatively: Take care of the patient, and show respect to the patient. Looking for opportunities to talk with the patient, but pay attention to the language, simple language is better. Repeat the chatting information if necessary. Through facial expressions, movements to express a variety of information, so that patients can feel the care nurses give to her. Play her favorite music for patient, and it will promote the recovery of consciousness.

Family assistants: It is necessary to teach patient's families how to do functional exercise for the patient, which can prevent muscle atrophy, joint deformity. Through the care measures above, the patient's muscle tension, facial expression improved a slight. Meanwhile, teach the family members about the knowledge of how to take good care of themselves when they look after the patient and must wash their hands after contact with the patient, thus, strengthen their self-protection awareness.

\section{Dealing with medical materials}

Instruments used on the patient was subjected to rigorous disinfection, instruments contacted to the patient need to soak into sodium hypochlorite solution for more than 60 minutes, and put into high-pressure steam sterilization after cleaning [11]. Disposable items used during the operation, and the patient's excretions, secretions, cerebrospinal fluid and brain tissue should be incinerated or buried [12]. Put clothing and bed sheet products into 132 degree pre-vacuum steam sterilization for at least 30 minutes.

\section{Conclusions}

In summary of this case, patient was found onset with cerebral infarction, a few weeks later the symptoms showed the signs of progressive dementia, myoclonus, with brain biopsy the patient was diagnosed with CJD. After the care measures above, the patient had no infection and complications. While in the nursing process it is important to minimize the suffering of the patient and improve the quality of life of the patient and prolong survival time. Health care workers need to strengthen self-protection strategies, and operation equipment and facilities must be strictly sterilized. Care education work for family members is equivalently necessary and important to avoid infection.

\section{References}

1. Creutzfeldt-Jakob Disease Surveillance Of And Other Diseases Prion.

2. Ying Li (2014) Based on the living conditions of rare disease research group - analysis on rare diseases and related policy development. J China Soft Science.

3. Jiang-jiang HE, Ya-bing Z (2012) The EU rare diseases Guarantee System and its Inspiration. J Chiinese Journal of Health Policy.

4. Yongwen Q, Jianqiang H, Xianxian Z (2001) 2 cases report of domestic mushroom umbrella occluder treatment ductus arteriosus. J ovale Second Military Medical University 21 : 495-496.

5. Biao C (1998) BSE and spongiform encephalopathy. J Chinese Journal of Neurology 31: 124.

6. Sujuan B (2013) probably Creutzfeldt encephalopathy infection protection management J. Nurses Training Magazine 28: 615-616.

7. Sperling R, Haak K, Hesson D, Hidde BB (2005) Creutzfeldt-Jakob Disease in the Obstetric patient. Journal of Obstetric, Gynecologic and Neonatal Nursing 34: 546-550. 
Citation: Feifei W (2016) A Case of Nursing Experience of Taking Care of Creutzfeldt-Jakob Disease Patient in China. J Nurs Care 5: 336. doi: 10.4172/2167-1168.1000336

Page 3 of 3

8. Bodenheimer T, Lorig K, Holman H, Grumbach K (2002) Patient selfmanagement of chronic disease in primary care. JAMA 288: 2469-2475.

9. Ciccone MM, Aquilino A, Cortese F, Scicchitano P, Sassara M, et al. (2010) Feasibility and effectiveness of a disease and care management model in the primary health care system for patients with heart failure and diabetes (Project Leonardo). Vasc Health Risk Manag 6: 297-305.

10. Meiling L, Qiuying Y, Songbin H (2011) A survey on patients' satisfaction of hospitalization after carrying out the demonstration project of Quality care activities. Journal of Nursing.
11. Yan H, Sugong M (2000) Iatrogenic Creutzfeldt-Jakob disease. Chinese Journal of Ecology 12: 1.

12. Ying T, Qi Y (2007) 2 cases of suspicious Creutzfeldt-Jakob disease care. Nursing Research 21: 836-837. 